\title{
Nuevas aportaciones a la lexicografía del anglicismo: una mirada al léxico erótico
}

FÉLIX RODRÍGUEZ GONZÁLEZ

Universidad de Alicante

frodriguez@ua.es

1. El sexo, y el amor y erotismo, con los que se entrelaza, probablemente sea el tema que más preocupa en nuestra cultura. Y es que el sexo es una dimensión vital de la existencia humana, el motor del mundo; no en vano a través de él se genera la vida. Sobre su temática a diario se vierten ríos de tinta, desde muy diferentes áreas y disciplinas, empezando por la biología y la medicina que nos recuerdan nuestra naturaleza animal. Pero la compleja condición humana requiere, además, un acercamiento desde muchos otros campos, como la psicología y psiquiatría, la sociología, la antropología cultural, la literatura, la política, la religión y la ética. Y enlazando con todos ellos, como herramienta indispensable, el lenguaje.

Sin embargo, a diferencia de lo ocurrido con los demás campos, al examinar el lenguaje del sexo se echa en falta una mayor dedicación por parte de los lingüistas, lo que se explica por varias razones. En primer lugar, por la propia materia, que, como queda dicho, se presta a múltiples dimensiones de análisis, y, además, contiene numerosas voces de argot y expresiones populares que forman una parte muy viva y en ebullición constante. En segundo lugar, no menos importante, por tratarse de un tema que continúa siendo tabú en nuestra sociedad, y esto exige buena dosis de apertura mental y valentía por parte del investigador. A pesar de que vivimos tiempos de mayor liberación en las costumbres, son muchas las personas que aún ven en el sexo algo intrínsecamente malo y peligroso, y su invocación les produce angustia y desasosiego, cuando no lo convierten en motivo de chanza.

El hecho de que haya un aluvión de términos referidos al sexo, en tanto que práctica o relación sexual, y renovados constantemente desde épocas inmemoriales, reflejan el peso del tabú que arrastran algunos de esos conceptos en nuestra cultura occidental, acrecentado en determinadas épocas como durante la dictadura. Y esto tanto en lo que atañe al ámbito homosexual como al heterosexual, aunque quizá sea más notorio en el homosexual. Se controlaba intencionadamente la expresión bien por pudor, o para sortear la crítica de los poderes públicos en determinados contextos sociales y políticos. Pero desde la Transición se asiste a un 'destape' también en la terminología sexual, y se hace gala a menudo de un lenguaje, más procaz, más directo, con un tono soez sin las cortapisas del pasado, y de ello dan buen testimonio la prensa periódica — por no 
hablar de las actuales redes sociales, no sometidas a control social- $\mathrm{y}$, finalmente, los que nos dedicamos a lexicografía, como notarios del habla cotidiana que debemos ser.

A ese 'destape' lingüístico ha contribuido de manera especial la influencia exterior, con el relato de nuevas costumbres, que de hecho arrancó a finales de los sesenta, por un lado con la llegada de la contracultura norteamericana que se hizo visible mayormente merced a las publicaciones marginales, y por otro por el 'boom' turístico, sobre todo de corte británico. Una influencia que desde entonces ha continuado sin cesar, llegando su punto álgido a finales del siglo xx, con la irrupción del internet, y a principios del XXI con las redes sociales, y en ambos casos abriéndose paso sin conocer fronteras. Y todo ello al abrigo de una divisa común, la lengua inglesa, a través de la cual nos ha llegado un aluvión de préstamos.

Los estudios sobre el anglicismo y el préstamo en general han ido creciendo a lo largo de los últimos decenios en numerosas lenguas, entre ellas la española, conforme el inglés se ha ido asentando por derecho propio como una lingua franca que favorece la comunicación internacional, a la vista del contexto de globalización económica y cultural en el que estamos inmersos (cf. Jenkins 2007). Con estas premisas en mente se comprende que la mayoría de los campos semánticos explorados, de manera parcial o monográfica, en tales investigaciones se hayan producido en campos especializados pero de interés general, como la economía, la tecnología, la industria, la informática, la comunicación audiovisual, la política, y cuyo léxico aflora con cierta frecuencia en publicaciones (diarios y revistas, mayormente) de corte generalista y en situaciones comunicativas dominadas por un registro formal. Menos se ha incidido, por el contrario, en áreas menos conocidas por el público general, bien por la limitación de su audiencia, como los lenguajes de ciertas subculturas, como las juveniles, o por su relación más o menos directa con la marginalidad, como es el caso de la drogadicción y el erotismo. De la presencia del anglicismo en el mundo de la droga ya di cuenta en varios estudios monográficos (Rodríguez González 1994), datos que podrían ampliarse con una mirada al propio diccionario referido a dicha especialidad (2014). En el caso de la sexualidad y el erotismo, concurre un factor adicional que a menudo disuade al investigador común, y es el propio carácter de su léxico, referido a conceptos que pueden ser familiares para algunos hablantes pero que, en todo caso, para la mayoría, son objeto de estigma social, como he señalado antes. Y, sin embargo, la influencia está ahí, a nada que se explore y se haga una incursión en este campo.

2. Un recuento del vocabulario de origen inglés dentro del lenguaje erótico de mi Diccionario del sexo y el erotismo [DSE] (2011), que constó de más de 6.200 entradas, arroja la cifra de 230, incluyendo en ellas solamente los anglicismos crudos, o directos, y los calcos y traducciones más visibles de los mismos (como salir del armario), así como algunas adaptaciones (wondercillo). Si a ese número añadimos los procedentes del Diccionario gay-lésbico (2008) y del reciente Gran diccionario de anglicismos (2017) no recogidos en el DSE, la suma llega a 250, es decir, alrededor del $4 \%$ del total, lo cual es una cifra estimable si se considera que se trata de un léxico muy especializado y restringido, y con un sabor mayormente informal y argótico. Presumible- 
mente la cifra sería muy superior si se considera la influencia en campos tan inabarcables como los de la ciencia y la tecnología.

Las motivaciones que subyacen a la acuñación y uso de este tipo de anglicismos son muy varias, como de hecho ocurre con los préstamos en general. Los hay que son clasificables como «denominativos», y por tanto necesarios, por referirse a

- nuevas realidades y conceptos, como los que designan artilugios o artefactos sexuales (andro-extender, butt plugs, dildo, glory hole, grope suit, pill card, prince Albert, sling o eslinga, tuppersex, wonderbra);

- nuevas prácticas o juegos sexuales, y sus participantes (barebacking, bamboning, bondage, bukkake, catfighting, docking, dogging, facesitting, felching, fistfucking (o fisting), furtling, gang-bang, rent-a-dread, sándwich, sex-boxing, sexting, sex party, shaving, show girl, spank, spanking, squeezing, strap-on, stretching, strip game, strip game, strip-tease o estriptís, stripper, table-girl, trampling, swinging, wraping);

- lugares, actividades o situaciones eróticas y sus participantes (body body, call girl, cruising, dark room, escort, flirt, fulmonty, gogo-girl, groupie, lovin-in, love hotel, night club, pijama party, petting, pin-up girl, playboy, playgirl, playmate, playroom, porno show, sexy boy, sexy girl, streaker, streaking, taxi-girl, tea-dance, topless, toplessera);

- nuevas identidades sexuales y subculturas (bi, bisex, dyke, gay, lesb, lesbi, lesbian chic, lesbo, queer, straight, transex, ubersexual; y nuevos subtipos: agrogay, auntie, autoreverse, cibergay, chubby, clon, bear, bear chaser, butch (y baby-butch), camp, daddy, femme, gayhetero (y galletero), gay power, gayskin, guppie, heterogay, homofreak, homosex, infragay, lesbigay, lipstick (y lipstic lesbian), luppie, maribuilding, macho gay, macho man, mariclón, Mari conan (o mariconan), marilycra, maripiercing, pink mafia, pink power, queen, dryke-friendly, gay friendly, heterofriendly);

- vestimenta erótica y sus usuarios: baby doll, baby top, chap, drag (dragformismo, dragmanía, draguerío), drag king (y king), drag queen (o dragcuin; draqueenismo, dragquiniano), dress code, hot pants, leather, leatheron y lederón, lederona, lederina, mini kilt, minishort, top model.

3. Frente a todos estos anglicismos, que cumplen una función «referencial» (o «ideacional», según la terminología de Halliday), están los que no aportan una noción nueva, o bien aportan un significado algo más específico, pero en cuyo uso prevalece en todo caso una finalidad «expresiva», positiva o negativa. Estas asociaciones son ambivalentes en la medida en que su carga emocional puede variar de signo según la condición del individuo, ya sea por su actitud o por el grupo social al que pertenece. El ejemplo más patente lo tenemos en la voz gay, que por primera vez, a finales de los años sesenta, en términos generales confirió una connotación ennoblecedora al antiguo 'marica', aunque puede conllevar también el significado específico de 'homosexual militante y orgulloso de serlo' y 'practicante de la homosexualidad'. En el lado contrario podríamos situar a lesbo, que puede ser utilizado con un matiz despectivo, sobre todo en círculos heterosexuales; y una connotación más neutra cabe presuponerse 
cuando se utiliza como forma combinatoria a modo de prefijo en diversos compuestos y derivados que hacen referencia a la 'lesbiana', siguiendo el modelo de homo- para 'homosexual' (lesbofobia, lesbofóbico, lesbofeminista, lesbofilia).

Buena parte de los neologismos en la esfera erótica en realidad cumplen una doble función, referencial y expresiva, por lo que me referiré a estos anglicismos con la etiqueta «denominativos-expresivos». Bien ilustrativos son aquellos que designan conceptos nuevos pero que cumplen una función eufemística y / o críptica y podrían calificarse como provocativos, como ocurre en el contexto de la prostitución y el negocio del sexo. Así, voces como blow job, bondage, call girl, caning, spanking, fistfucking aparecen en la prensa española, dentro de anuncios de contactos, y el carácter extranjero ablanda u oculta la crudeza del concepto. ¿Puede imaginarse por un momento la reacción del lector de un diario generalista de gran tirada, como El País o El Mundo, y no digamos de uno con un tinte más conservador, si en lugar de fistfucking, o sus derivados fistear, fister, fistero, fisting, se encontrara con la versión en español castizo de tales expresiones? Claro que tratándose de ocupaciones y actividades socialmente estigmatizadas, estos anglicismos, en sus formas directas y patentes, se convierten en índices léxicos de cohesión e identidad grupal, lo que explica que algunos de ellos terminen formando parte de una terminología internacional.

Muy novedosas son también las recientes creaciones periodísticas sugar baby, sugar daddy, sugar mommy, toy boy, todas ellas referentes a la unión contractual con carácter temporal de dos personas de diferente edad y condición que intercambian belleza y juventud por el estatus de 'mantenidas' o 'acompañantes', normalmente con relación sexual de por medio, con su consiguiente lucro económico y bienestar.

El eufemismo y el disfemismo pueden aplicarse igualmente a un concepto de uso más ordinario y conversacional, como MILF (o milf), procedente de la expresión inglesa Mom I'd Like to Fuck ('madre que me gustaría follar') ${ }^{1}$ que, envuelto bajo el manto de una sigla, ha resultado admisible por la audiencia lectora pero cuyo significante analizado en su literalidad resulta ofensivo por su procacidad. Y sorprendentemente ha cuajado por su ingeniosidad y necesidad expresiva, pues en unos tiempos donde la esperanza de vida ha aumentado y la cosmética ha ayudado a mejorar el 'look' de las personas, es fácil pensar que voces como cincuentona o sesentona, para una mujer madura atractiva y con apariencia 'sexy' quedaban fuera de lugar. El término es ilustrativo de lo que podríamos llamar un «doble eufemismo», por un lado el primario y básico, que por su condición de extranjería oculta lo que en realidad, en sentido estricto, es un «disfemismo» ${ }^{2}$, al que se superpone el carácter abreviativo, que contribuye a opacar aún más el significado de su forma plena al hablante no familiarizado con el inglés. En estas circunstancias, queda abierta la vía para adquirir estatus de ciudadanía léxica, lo

\footnotetext{
${ }^{1} \mathrm{Su}$ equivalente en español americano es $M Q M C$ ('madre que me cogería'). Coger es un vulgarismo para 'follar' en algunas variedades del otro lado del Atlántico, como en el español de Argentina, polisemia que es fuente de más de un chiste cuando se cruzan hablantes de culturas tan dispares.

2 Sobre los conceptos de eufemismo y disfemismo y sus sutiles diferencias, cf. CrespoFernández (2015: 45-50).
} 
queda demostrado cuando encontramos milf, escrito en redonda, y en sentido figurado aplicado a la ciudad de Barcelona, como revelan estos textos:

Barcelona hoy está buena. Muy buena. En términos heterosexuales, es ya una MILF de pleno derecho. (Risto Mejide, «Barcelona, 'pensa't' guapa», El Periódico Extremadura, 02/02/2014)

Un traje sobre el que nuestro paparazzo no se abstuvo de opinar en un tuit subsiguiente, gimiendo Oh my god! como una milf sajona pillada en el acto. (Arcadi Espada, «O llevaré burka por ti», El Mundo, 30/05/2015)

También de uso conversacional entre los jóvenes y con aplicación general es petting, que en parte viene sustituyendo a voces de sabor anticuado como magreo, manoseo, meter mano, pero que en realidad son sinónimos parciales. El anglicismo adquiere un toque de modernidad y se convierte además en una especie de hiperónimo con un significado más específico al englobar todo que no pueda definirse como el acto sexual propiamente dicho.

4. Al lado de las funciones «referencial»y «expresiva», y no siempre en oposición, está la «textual», bajo la que se subsumen diferentes motivaciones. Por un lado, la «precisión significativa»y la «claridad» que revelan anglicismos como los que se han comentado (gay, petting...), u otros como single o neosoltero, sex-store y sex-shop, al lado de su traducción tienda erótica y la formación híbrida sex-tienda, night-club frente a club nocturno; la «economía de expresión» (gay frente a homosexual), unida a un toque de humor como en jet (igualmente 'homosexual [masculino]), pink (adj. 'homosexual'), kiki (del inglés quickie / polvo rápido, rapidito), swinging (intercambio de parejas); y la «variación estilística», pues en ciertos casos la elección del anglicismo responde a la búsqueda de una forma sinónima alternante como recurso co-referencial para evitar repeticiones en un texto, sobre todo escrito: claros ejemplos son los de gays / homosexuales, top models / modelos, a los que se podría añadir otros muchos, como cross dresser / travesti, cross dressing / travestismo, cougar (woman) / (mujer) pantera, fast sex / sexo rápido, slow sex / sexo lento, love-affair / aventura amorosa, glory hole / agujero glorioso, multiloving / poliamor, pornostar / estrella porno, rimming / beso negro. Y el hablar de estilo implica que la elección es principalmente idiolectal, como una opción dependiente sobre todo del usuario, mayormente escritor (cf. Rodríguez González 1996).

Capítulo aparte son las innovaciones fraseológicas procedentes de calcos o traducciones que por motivos similares irrumpen en nuestro idioma. En casos como salir del armario ( $<$ to come out of the closet), salida del armario ( $<$ coming out of the closet), cuarto oscuro ( $<$ dark room), follamigo ( $<$ fuckfriend), follamadres $(<$ motherfucker), hotel de amor (< love hotel), lluvia dorada (< golden shower), se introduce una idea nueva, un concepto específico. En otros, como tener sexo $(<$ to have sex) y hacer el amor (< to make love), la motivación es puramente eufemística pues evitan formas alternativas castizas más directas y vulgares. La última de estas, hacer el amor, supuso una modificación de sentido frente al uso anterior, basado en el étimo francés faire l'amour, que desde muy antiguo fue portador del significado de 'cortejar', 'lisonjear'. 
Podría añadirse en esta línea una formación compuesta muy singular utilizada en el léxico lésbico, mariliendre(s), que ha sido inducida a partir del inglés, fag hag (de fag 'maricón' y hag 'vieja bruja'), empleado para referirse a la 'mujer heterosexual que busca compañía de hombres gays por los lugares de ambiente'. El término fue acuñado por el escritor Luis Antonio de Villena, posteriormente divulgado con cierto éxito por el escritor Leopoldo Alas, y es una creación humorística en la que se ha recurrido ingeniosamente por vía metafórica al cruce de María (típico nombre de mujer) y liendre (huevo del piojo), es decir, mujer que se pega como un piojo, como un parásito. Y el piojo produce molestias con sus picaduras $(D G L)$.

Para terminar este repaso al léxico erótico cabe mencionar el uso del inglés para denominaciones de portales de contactos empleados con creciente frecuencia en España por el ingente número de solteros o «singles», jóvenes y mayores, como e-darling, meetic, tinder, como recurso de citas rápidas y anónimas, fenómeno favorecido por el auge de las redes sociales y las nuevas tecnologías que han arrinconado las antiguas formas de relación (presentaciones, baile, tasqueo, etc.). Las alusiones son muy significativas; así, e-darling significa 'querido electrónico', meetic evoca meeting 'encuentro', y tinder 'yesca', esto es, materia seca que puede arder con una simple chispa, y por extensión, cualquier cosa que excita las pasiones.

\section{REFLEXIONES FINALES Y CONCLUSIÓN}

El vocabulario erótico de carácter anglicista constituye un subsistema léxico muy singular debido a su particular multifuncionalidad, dado que buena parte de sus voces son susceptibles de cumplir funciones lingüísticas varias al mismo tiempo. La más evidente es la «referencial», al contribuir el anglicismo a menudo a llenar un «hueco ecológico» en la lengua, pero a veces se emplea en un determinado contexto por mor de una mayor precisión significativa, y una connotación positiva y de modernidad, y una economía lingüística, e incluso por variación estilística. De todo ello da testimonio, como hemos señalado, gay, sin duda el término más clarificador. Pero los ejemplos podrían repetirse.

Me permitiré añadir uno más, muy reciente, y que resulta igualmente iluminador, single, dentro de un campo léxico más abierto, si se quiere, pero lindante igualmente con la sexualidad. Hace referencia a una persona soltera que disfruta y se siente orgullosa de su condición, un concepto para el que la lengua española esporádicamente emplea como sinónimo neosoltero. Está tomado directamente del inglés y ha adquirido una connotación positiva que no tenía soltero, al igual que ocurriera con el préstamo de gay frente al de homosexual. Soltero connota soledad, tristeza, fracaso, pues apunta a un ser solitario, aislado, incompleto, que permanece pasivamente a la espera de una oportunidad — su «media naranja»—, para abandonar el estado de soltería. Single, en cambio, connota individualidad, autonomía, independencia, pero también disfrute de la vida, lo que entraña, por tanto, una actitud más positiva y abierta en la búsqueda de nuevas relaciones, orientada hacia la amistad y no necesariamente hacia el amor. Con ello se ahuyenta el tabú que venía arrastrando la palabra solterona (más que solterón); ahora hay una reivindicación de la vida en solitario, o mejor, «en singular» (precisamente 'singular' es otro de los significados que la palabra tiene en inglés). Por otro 
lado, la diferencia que separa a ambos términos no es sólo la connotación, también el contenido. El soltero no se ha casado nunca, el single está solo, sin pareja, y engloba por tanto al soltero o no casado, pero también al viudo, divorciado y separado; o, de forma conjunta, al que está «libre de amores» (fancy-free, dicen en argot inglés ${ }^{3}$, y de forma más enfática footloose and fancy-free 'libre como el viento', dando a entender que uno puede hacer lo que quiera, y en un estilo más formal, y más neutro, nonattached 'sin ataduras, sin compromiso'). Algunos extienden el significado para referirse incluso al que tiene pareja pero comparte una filosofía «single».

En las grandes ciudades de los países de habla inglesa, como Estados Unidos y el Reino Unido, hasta hace poco existían los singles'bars ('bares de solteros'), donde se daban cita las personas que estaban libres, sin pareja, institución que ha sido arrinconada ante las posibilidades abiertas por Internet, lo que no quiere decir — más bien, al contrario- que no se propicien encuentros organizados y fiestas para congregar a las personas que se encuentran en tal situación y con ánimo de divertirse, idea que en los últimos años viene siendo bien acogida también en España.

Con estos y otros muchos ejemplos de anglicismos que podrían aportarse dentro del campo del erotismo y la sexualidad, comprobamos que con frecuencia las funciones lingüísticas tal como se describen en la sincronía de la lengua no son compartimentos estancos, sino que se entrecruzan debido a las distintas motivaciones que respaldan su uso.

Una segunda reflexión se impone a propósito de la semántica de estos anglicismos con referentes eróticos. A pesar de los cambios sociológicos producidos en esta área en las últimas décadas, con una mayor liberalidad en las costumbres, el eufemismo (y el disfemismo) constituye un recurso de primer orden a la hora de explicar los neologismos, tanto de forma como de sentido, que se abren paso de forma constante y que son un buen exponente de la capacidad revitalizadora de la lengua. $Y$ el anglicismo, por sus propiedades lingüísticas y su morfología, ofrece unas posibilidades añadidas a las que ostentan otras expresiones de nuestro léxico.

Conviene señalar, además, que en algunos anglicismos que irrumpen en la esfera sexual, tanto si se trata de eufemismos como de disfemismos propiamente dichos, hay un componente metafórico que les confiere un carácter marcadamente humorístico y argótico. Así, cougar (o su calco pantera) se incluye en la metáfora conceptual LA MUJER ES UN ANIMAL SALVAJE, que sugiere la idea de una mujer depredadora a la búsqueda de hombres para saciar su apetito sexual; sugar baby cosifica a una joven como un caramelo con el que endulzar la vida de un señor mayor en virtud de la metáfora LAS MUJERES SON DULCES; y toy boy materializa la conceptualización EL COMPAÑERO SEXUAL ES UN JUGUETE que convierte a un joven en juguete a disposición de la mujer (u hombre, en una relación homosexual) que lo mantiene. Aún más plástica es la metáfora del closet (armario o ropero) que encierra a la persona homosexual que se resiste a revelar su identidad en la sociedad actual, con sus múltiples formaciones derivadas

${ }^{3}$ La frase fue acuñada por William Shakespeare en A Midsummer Nights Dream (c1595). 
closetero, closeteado, enclosetado, en varios países de la América, y armariado, armarizado, armarizar, aramarización en el español peninsular (DGL; cf. CrespoFernández 2015: 107-109; Crespo-Fernández y Luján-García 2017).

\section{REFERENCIAS BIBLIOGRÁFICAS}

CRESPO-FernÁndeZ, Eliecer (2015): Sex in Language Euphemistic and Dysphemistic Metaphors in Internet forums, Londres, Bloomsbury.

CRESPO-FERNÁNDEZ, Eliecer y Carmen LuJÁN-GARCÍA (2013): «Anglicismo y tabú: Los valores axiológicos del anglicismo crudo en la esfera del erotismo y la sexualidad», Revista de Estudios Filológicos, 52, pp. 53-74.

CRESPO-FERNÁNDEZ, Eliecer y Carmen LuJÁN-GARCía (2017): «Anglicism and Word Axiology in Homosexual Language», RESLA, 30, 1, pp. 74-103.

CRESPO-FERNÁNDEZ, Eliecer y Carmen LuJÁN-GARCÍA (2018): Anglicismos sexuales en español: el inglés como recurso eufemístico y disfemístico en la comunicación virtual, Granada, Comares.

JENKINS, Jennifer (2007): English as a Lingua Franca: Attitude and Identity, Oxford, Oxford University Press.

RodríGuez GonZÁlez, Félix (1994): «Anglicismos en el argot de la droga», Atlantis, 16, 1/2, pp. $179-216$.

RODRÍGUEZ GONZÁLEZ, Félix. (1994): «Remarques sur les glissements de sens dans l'argot. A propos du langage de la drogue et les anglicismes en espagnol», Cahiers de Lexicologie, 64, 1, pp.147-154.

RodríGUEZ GONZÁLEZ, Félix (1994) «English-Spanish Glossary of Drug-Related Slang», Lebende Sprachen, 39, 3, pp. 123-124.

RODRÍGUEZ GONZÁLEZ, Félix (1996): «Functions of anglicisms in contemporary Spanish», Cahiers de lexicologie, 68, 1, pp. 107-128.

RodríGuez GonZÁlez, Félix (2008): Diccionario gay-lésbico. Vocabulario general y argot de la homosexualidad [DGL], Madrid, Gredos.

RodríGUEZ GONZÁLEZ, Félix (2011): Diccionario del sexo y del erotismo [DSE], Madrid, Alianza.

Rodríguez GONZÁLEZ, Félix (2014): Diccionario de la droga: vocabulario técnico y argot, Madrid, Arco/Libros.

RODRíGUEZ GONZÁLEZ, Félix (2017): Gran diccionario de anglicismos, Madrid, Arco/Libros.

\section{Título/TitLe}

«Nuevas aportaciones a la lexicografía del anglicismo: una mirada al léxico erótico» «New contributions to the lexicography of anglicisms: a look at the erotic language» 
Resumen: El trabajo examina la enorme influencia del inglés registrada en el ámbito del sexo y el erotismo en las últimas décadas, especialmente a partir de la Transición, al compás de los cambios sociales y políticos y los nuevos aires de libertad que han tenido lugar en España. En particular, pasa revista a los anglicismos léxicos y calcos que han ido poblando los distintos campos semánticos que conforman esta esfera del lenguaje (artilugios y juegos sexuales, vestimenta erótica, nuevas identidades sexuales, etc.).

Palabras clave: anglicismo; préstamos de palabras; lenguas en contacto; léxico; lexicografía.

Abstract: The aim of this article is to examine the enormous influence of English in the field of sex and eroticism in Spanish in the last decades, especially after the Transition period, keeping pace with the rapid social and political changes and the new airs of freedom which have taken place in Spain. In particular, it reviews the lexical anglicisms and calques that have filled the various semantic fields conforming this sphere of language (sexual devices and games, erotic dresses, new sexual identities, etc.).

Key words: anglicisms; borrowings; contact linguistics; lexis; lexicography. 